\title{
OPE and the NLL-resummed evolution for B-meson distribution amplitude: radiative and nonperturbative corrections to inverse moments for exclusive $B$ decays
}

\author{
Hiroyuki Kawamura* \\ Department of Mathematical Sciences, University of Liverpool, Liverpool, L69 3BX, United \\ Kingdom \\ E-mail: Hiroyuki.Kawamura@liverpool.ac.uk
}

\section{Kazuhiro Tanaka ${ }^{\dagger}$}

Department of Physics, Juntendo University, Inba, Chiba 270-1695, Japan

E-mail: tanakak@sakura.juntendo.ac.jp

\begin{abstract}
The $B$-meson distribution amplitude (DA) is defined as the matrix element of a quark-antiquark bilocal light-cone operator, associated with a long-distance component in QCD factorization formula for exclusive $B$ decays. We treat the corresponding bilocal operator based on the operator product expansion (OPE), as the short-distance expansion for the quark-antiquark light-like distance $t$. The matching at the next-to-leading order $\alpha_{s}$ with the local operators of dimension $d \leq 5$ manifests the OPE that has the Wilson coefficients, receiving the cusp singularity, and the novel higher-dimensional operators with additional gluons. This OPE is useful for a low renormalization scale $\mu \sim 1 \mathrm{GeV}$, allowing us to derive the model-independent form of the $B$-meson DA for $t \lesssim 1 / \mu$ in terms of a few nonperturbative parameters. We also discuss the evolution of the DA, which is governed by the cusp as well as the DGLAP-type anomalous dimension. These anomalous dimensions give the "quasilocal" kernel in the coordinate-space representation, and the corresponding evolution equation can be solved analytically, accomplishing the relevant Sudakov resummation. This result allows us to calculate the $B$-meson DA at the factorization scale $\sqrt{m_{b} \Lambda_{\mathrm{QCD}}}$ using our OPE-based DA as the input at $\mu \sim 1 \mathrm{GeV}$. The impact of our results on the integral relevant to exclusive $B$ decays, $\lambda_{B}$, is discussed.
\end{abstract}

RADCOR 2009 - 9th International Symposium on Radiative Corrections (Applications of Quantum Field Theory to Phenomenology)

October 25-30 2009

Ascona, Switzerland

${ }^{*}$ Supported in part by the UK Science \& Technology Facilities Council under grant number PP/E007414/1. † Speaker. 
For the exclusive $B$-meson decays, such as $B \rightarrow \pi \pi, \rho \gamma, \ldots$, systematic methods have been developed using QCD factorization based on the heavy-quark limit [1,2]. In the corresponding factorization formula of the decay amplitude, essential roles are played by the light-cone distribution amplitudes (LCDAs) for the participating mesons, which include nonperturbative long-distance contributions. In particular, in addition to the LCDAs for the light mesons $\pi, \rho$, etc., produced in the final state, the LCDA $\tilde{\phi}_{+}$for the $B$ meson, defined as the vacuum-to-meson matrix element [3],

$$
\tilde{\phi}_{+}(t, \mu)=\frac{1}{i F(\mu)}\left\langle 0\left|\bar{q}(t n) \mathrm{P} e^{i g \int_{0}^{t} d \lambda n \cdot A(\lambda n)} h \gamma_{5} h_{v}(0)\right| \bar{B}(v)\right\rangle=\int d \omega e^{-i \omega t} \phi_{+}(\omega, \mu),
$$

participates in the processes where a large momentum is transferred to the spectator light-quark via gluon exchange [2]. Here, the bilocal operator is built of the $b$-quark and light-antiquark fields, $h_{v}(0)$ and $\bar{q}(t n)$, linked by the Wilson line at a light-like separation $t n$, with $n_{\mu}$ as the light-like vector $\left(n^{2}=0, n \cdot v=1\right)$, and $v_{\mu}$ representing the 4-velocity of the $B$ meson; a difference between (1) and the familiar pion-LCDA is that $h_{v}(0)$ is an effective field in the heavy-quark effective theory (HQET). $\mu$ denotes the scale where the operator is renormalized, and $F(\mu)$ is the decay constant in HQET, $F(\mu)=-i\left\langle 0\left|\bar{q} h \gamma_{5} h_{v}\right| \bar{B}(v)\right\rangle$. The RHS in (1) defines the momentum representation, with $\omega v^{+}$denoting the light-cone component of the momentum of the light antiquark.

The "IR structure" of (1), studied using constraints from the equations of motion (EOM) and heavy-quark symmetry [3], as well as the "UV structure", which was calculated in the 1-loop renormalization of the bilocal operator in (1) $[4,5]$, is notoriously peculiar compared with the pion LCDA: the IR structure of (1) receives the complicated mixing of the multiparticle Fock states of higher-twist nature through the nonperturbative quark-gluon interactions. On the other hand, the UV structure indicates that the radiative corrections yield the contributions that are singular as $t \rightarrow$ 0 , so that the bilocal operator of (1) is not Taylor expandable about $t=0$. In such circumstance, it is the operator product expansion (OPE), $\bar{q}(t n) \mathrm{P} e^{j g \int_{0}^{t} d \lambda n \cdot A(\lambda n)} h h \gamma_{5} h_{v}(0) \sim \sum_{i} C_{i}(t, \mu) \mathscr{O}_{i}(\mu)$ as $t \rightarrow 0$, that enables us to treat both the UV and IR behaviors simultaneously: all the singular contributions are absorbed into the coefficient functions $C_{i}(t, \mu)$, while the multiparticle Fock states are embodied by the higher-dimensional quark-gluon operators arising in the local composite operators $\mathscr{Q}(\mu)$. A first systematic treatment of the mixing of the multiparticle operators, disentangling the (singular) radiative corrections as the coefficients, has recently been accomplished by us [6], yielding

$$
\bar{q}(t n) \mathrm{P} e^{i g \int_{0}^{t} d \lambda n \cdot A(\lambda n)} h \gamma_{5} h_{v}(0) \sim C_{1}^{(3)}(t, \mu) \mathscr{O}_{1}^{(3)}(\mu)+\sum_{k=1}^{2} C_{k}^{(4)}(t, \mu) \mathscr{O}_{k}^{(4)}(\mu)+\sum_{k=1}^{7} C_{k}^{(5)}(t, \mu) \mathscr{O}_{k}^{(5)}(\mu)
$$

where the summation is over a basis of local operators of dimension- $d, \mathscr{O}_{k}^{(d)}(k=1,2, \ldots)$, defined as $\mathscr{O}_{1}^{(3)} \equiv \bar{q} h \gamma_{5} h_{v},\left\{\mathscr{O}_{k}^{(4)}\right\} \equiv\left\{\bar{q}(i n \cdot \overleftarrow{D}) \not h \gamma_{5} h_{v}, \bar{q}(i v \cdot \overleftarrow{D}) h h \gamma_{5} h_{v}\right\}$, and $\left\{\mathscr{O}_{k}^{(5)}\right\} \equiv\left\{\bar{q}(i n \cdot \overleftarrow{D})^{2} \not h \gamma_{5} h_{v}\right.$, $\bar{q}(i v \cdot \overleftarrow{D})(i n \cdot \overleftarrow{D}) h \gamma_{5} h_{v}, \bar{q}(i v \cdot \overleftarrow{D})^{2} \not h \gamma_{5} h_{v}, \bar{q} i g G_{\alpha \beta} v^{\alpha} n^{\beta} \not h \gamma_{5} h_{v}, \bar{q} i g G_{\alpha \beta} \gamma^{\alpha} n^{\beta} \not \vec{h} \gamma_{5} h_{v}, \bar{q} i g G_{\alpha \beta} \gamma^{\alpha} v^{\beta} \not h \gamma_{5} h_{v}$, $\left.\bar{q} g G_{\alpha \beta} \sigma^{\alpha \beta} \not h \gamma_{5} h_{v}\right\}$, with another light-like vector, $\bar{n}^{2}=0$, as $v_{\mu}=\left(n_{\mu}+\bar{n}_{\mu}\right) / 2$, and $G_{\alpha \beta}$ being the gluon field strength tensor. The Wilson coefficients are obtained at the NLO in the $\overline{\mathrm{MS}}$ scheme, as $C_{1}^{(3)}(t, \mu)=1-\left(\alpha_{s} C_{F} / 4 \pi\right)\left(2 L^{2}+2 L+5 \pi^{2} / 12\right), C_{1}^{(4)}(t, \mu)=-i t\left[C_{1}^{(3)}(t, \mu)+\alpha_{s} C_{F} L / 4 \pi\right], C_{2}^{(4)}(t, \mu)$ $=\left(i t \alpha_{s} C_{F} / 4 \pi\right)(4 L-3), C_{1}^{(5)}(t, \mu)=-t^{2}\left[C_{1}^{(3)}(t, \mu)+\alpha_{s} C_{F} L / 3 \pi\right] / 2$, where $C_{F}=\left(N_{c}^{2}-1\right) /\left(2 N_{c}\right)$, $\alpha_{s} \equiv \alpha_{s}(\mu)$, and $L=\ln \left[i(t-i 0) \mu e^{\gamma_{E}}\right]$ with the Euler constant $\gamma_{E}$; for the explicit form of $C_{2}^{(5)}(t, \mu)$, $C_{3}^{(5)}(t, \mu), \ldots, C_{7}^{(5)}(t, \mu)$, we refer the readers to [6]. In the corresponding matching calculation involving the three-body operators $\mathscr{O}_{4,5,6,7}^{(5)}$ of dimension 5 , we calculate the 3-point as well as 2-point 
function with the insertion of the bilocal operator in (1), where the diagrams for the former are obtained by attaching the external gluon line to those for the latter in all possible ways. We find that the 1-loop corrections for the 2-point function induce all of the above ten local operators using the $\mathrm{EOM}$, and, in particular, the double logarithm $L^{2}$ in the corresponding coefficient functions originates from the vertex-type correction around a "cusp" between the two Wilson lines, the light-like Wilson line of (1) and the time-like one from $h_{v}(0)=\mathrm{P} \exp \left[i g \int_{-\infty}^{0} d \lambda v \cdot A(\lambda v)\right] h_{v}(-\infty v)$. The 3point function induces only $\mathscr{O}_{4,5,6,7}^{(5)}$ associated with the field-strength tensor, so that $C_{4,5,6,7}^{(5)}(t, \mu)$ involve the color factor $C_{G}=N_{c}$ as well as $C_{F}$ [6]. Substituting (2) into (1), we obtain [6],

$$
\begin{gathered}
\tilde{\phi}_{+}^{\mathrm{OPE}}(t, \mu)=1-\frac{\alpha_{s} C_{F}}{4 \pi}\left(2 L^{2}+2 L+\frac{5 \pi^{2}}{12}\right)-i t \frac{4 \bar{\Lambda}}{3}\left[1-\frac{\alpha_{s} C_{F}}{4 \pi}\left(2 L^{2}+4 L-\frac{9}{4}+\frac{5 \pi^{2}}{12}\right)\right] \\
-t^{2} \bar{\Lambda}^{2}\left[1-\frac{\alpha_{s} C_{F}}{4 \pi}\left(2 L^{2}+\frac{16}{3} L-\frac{35}{9}+\frac{5 \pi^{2}}{12}\right)\right]-\frac{t^{2} \lambda_{E}^{2}(\mu)}{3}\left[1-\frac{\alpha_{s} C_{F}}{4 \pi}\left(2 L^{2}+2 L-\frac{2}{3}+\frac{5 \pi^{2}}{12}\right)\right. \\
\left.+\frac{\alpha_{s} C_{G}}{4 \pi}\left(\frac{3}{4} L-\frac{1}{2}\right)\right]-\frac{t^{2} \lambda_{H}^{2}(\mu)}{6}\left[1-\frac{\alpha_{s} C_{F}}{4 \pi}\left(2 L^{2}+\frac{2}{3}+\frac{5 \pi^{2}}{12}\right)-\frac{\alpha_{s} C_{G}}{8 \pi}(L-1)\right] .
\end{gathered}
$$

This OPE form enables us to evaluate the $B$-meson LCDA for interquark distances $t$ with $t \lesssim 1 / \mu$ in a rigorous way in terms of three nonperturbative parameters in the HQET, which participate in the matrix element $\langle\cdots\rangle \equiv\langle 0|\cdots| \bar{B}(v)\rangle$ of the local operators in (2) through the use of the EOM and heavy-quark symmetry (see [3]): $\left\langle\mathscr{O}_{1}^{(4)}\right\rangle=4 i F(\mu) \bar{\Lambda} / 3,\left\langle\mathscr{O}_{2}^{(4)}\right\rangle=i F(\mu) \bar{\Lambda}$, with $\bar{\Lambda}=m_{B}-m_{b}$ being the mass difference between the $B$-meson and $b$-quark, and all seven matrix elements $\left\langle\sigma_{k}^{(5)}\right\rangle$ of dimension-5 operators can be expressed by $F, \bar{\Lambda}$ and two additional HQET parameters $\lambda_{E}$ and $\lambda_{H}$, which are associated with the chromoelectric and chromomagnetic fields inside the $B$ meson as $\left\langle\bar{q} g \boldsymbol{E} \cdot \boldsymbol{\alpha} \gamma_{5} h_{v}\right\rangle=F(\mu) \lambda_{E}^{2}(\mu)$ and $\left\langle\bar{q} g \boldsymbol{H} \cdot \boldsymbol{\sigma} \gamma_{5} h_{v}\right\rangle=i F(\mu) \lambda_{H}^{2}(\mu)$, respectively, in the rest frame.

The UV structure embodied in (2), (3) determines the scale dependence of the $B$-meson LCDA (1). Taking the derivative of (3) with respect to $\mu$ and combining with the scale dependence of the HQET parameters, i.e., $d \bar{\Lambda} / d \mu=0$ and that of $\lambda_{E, H}^{2}(\mu)$ (see [6]), we find [9]

$$
\mu \frac{d}{d \mu} \tilde{\phi}_{+}(t, \mu)=-\left[\Gamma_{\text {cusp }}\left(\alpha_{s}\right) L+\gamma_{F}\left(\alpha_{s}\right)\right] \tilde{\phi}_{+}(t, \mu)+\int_{0}^{1} d z K\left(z, \alpha_{s}\right) \tilde{\phi}_{+}(z t, \mu),
$$

where $\Gamma_{\text {cusp }}\left(\alpha_{s}\right)=\left(\alpha_{S} / 4 \pi\right) \Gamma_{\text {cusp }}^{(1)}$, and similarly for $\gamma_{F}\left(\alpha_{s}\right)$ and $K\left(z, \alpha_{s}\right)$, with $\Gamma_{\text {cusp }}^{(1)}=4 C_{F}, \gamma_{F}^{(1)}=$ $2 C_{F}$, and $K^{(1)}(z)=4 C_{F}(z /(1-z))_{+}$. The derivative of the "universal" double logarithmic term, $-\left(\alpha_{s} C_{F} / 4 \pi\right) 2 L^{2}$, in (3) yields the term associated with $\Gamma_{\text {cusp }}\left(\alpha_{s}\right)$ in (4). Indeed, (4) can be obtained by calculating the renormalization constant for the bilocal operator in (1), and $\Gamma_{\text {cusp }}\left(\alpha_{s}\right)$ corresponds to the anomalous dimension of the Wilson line with a cusp (see the discussion above (3)); $\gamma_{F}\left(\alpha_{s}\right)$ represents the anomalous dimension from the two quark fields in (1), combined with the "hybrid" anomalous dimension that governs the scale dependence of the decay constant $F(\mu)$; $K\left(z, \alpha_{s}\right)$ involves the "plus"-distribution as the DGLAP-type splitting function that originates from the vertex-type correction diagram associated with the massless degrees of freedom only. It is a remarkable property in (4) that the evolution kernel is quasilocal, such that the evolution mixes the LCDA with itself and with the LCDA associated with smaller light-cone separation (see also [7]): Fourier transforming (4) to the momentum space, we derive the evolution equation for $\phi_{4}(\omega, \mu)$ of (1), and reproduce the result obtained in [4], which mixes $\phi_{+}(\omega, \mu)$ with $\phi_{+}\left(\omega^{\prime}, \mu\right)$ over the entire 
region, $0<\omega / \omega^{\prime}<\infty[4,8]$. In contrast to the case of the DAs for the light mesons, we cannot derive the moment-space representation of (4), because the presence of $L$ prevents us from performing the Taylor expansion about $t=0$. Thus, the evolution equation for the $B$-meson LCDA manifests simple geometrical structure as the quasilocality only in the coordinate-space representation (4).

We are able to find exact analytic solution for (4) as [9] ( $\mu_{0}$ denotes an initial scale)

$$
\tilde{\phi}_{+}(t, \mu)=e^{\mathscr{V}\left(\mu, \mu_{0}\right)}\left(\tau \mu_{0} e^{\gamma_{E}}\right)^{-\xi} \frac{e^{\left(1-\gamma_{E}\right) \xi}}{\Gamma(\xi)} \int_{0}^{1} d z\left(\frac{z}{1-z}\right)^{1-\xi} \tilde{\phi}_{+}\left(z t, \mu_{0}\right),
$$

where $(z /(1-z))^{1-\xi}$ corresponds to the renormalization-group improvement of the splitting function $(z /(1-z))_{+}$in $(4)$, and $\left(\beta\left(\alpha_{s}\right)=\mu d \alpha_{s} / d \mu\right.$ is the $\beta$ function)

$$
\xi=\int_{\alpha_{s}\left(\mu_{0}\right)}^{\alpha_{s}(\mu)} \frac{\Gamma_{\text {cusp }}(\alpha)}{\beta(\alpha)} d \alpha, \quad \mathscr{V}\left(\mu, \mu_{0}\right)=-\int_{\alpha_{s}\left(\mu_{0}\right)}^{\alpha_{s}(\mu)} \frac{d \alpha}{\beta(\alpha)}\left[\Gamma_{\text {cusp }}(\alpha) \int_{\alpha_{s}\left(\mu_{0}\right)}^{\alpha} \frac{d \alpha^{\prime}}{\beta\left(\alpha^{\prime}\right)}+\gamma_{F}(\alpha)\right] .
$$

Fourier transforming (5), we reproduce the evolution derived in the momentum space in $[4,8]$, and recognize that our coordinate-space solution (5), inheriting the quasilocal structure from (4), provides the most compact expression possible for calculating the evolution of the $B$-meson LCDA. It is straightforward to see that the first two factors in (5), $e^{\mathscr{Y}\left(\mu, \mu_{0}\right)}\left(\tau \mu_{0} e^{\gamma_{E}}\right)^{-\xi}$, with (6) are exact even when the higher-order terms in $\alpha_{s}$ are included in $\Gamma_{\text {cusp }}\left(\alpha_{s}\right)$ and $\gamma_{F}\left(\alpha_{s}\right)$, representing the general solution of (4) when $K\left(z, \alpha_{s}\right) \rightarrow 0$. Thus, we extend (4)-(6) by including the 2-loop cusp anomalous dimension as $\Gamma_{\text {cusp }}\left(\alpha_{s}\right) \rightarrow\left(\alpha_{s} / 4 \pi\right) \Gamma_{\text {cusp }}^{(1)}+\left(\alpha_{s} / 4 \pi\right)^{2} \Gamma_{\text {cusp }}^{(2)}$, with $\Gamma_{\text {cusp }}^{(2)}=4 C_{F}[(67 / 9-$ $\left.\left.\pi^{2} / 3\right) C_{G}-10 N_{f} / 9\right]$, where $N_{f}$ denotes the number of flavors; this is useful for accomplishing the relevant Sudakov resummation: (6) is explicitly given by $\xi=\left(\Gamma_{\text {cusp }}^{(1)} / 2 \beta_{0}\right) Y+\cdots$, and

$$
\begin{aligned}
\mathscr{V}\left(\mu, \mu_{0}\right)= & \frac{\Gamma_{\text {cusp }}^{(1)}}{4 \beta_{0}^{2}}\left\{\frac{4 \pi}{\alpha_{s}\left(\mu_{0}\right)}(1+Y)-\frac{4 \pi}{\alpha_{s}(\mu)}\right\}-\frac{\gamma_{F}^{(1)}}{2 \beta_{0}} Y \\
& +\frac{\Gamma_{\text {cusp }}^{(1)}}{4 \beta_{0}^{2}}\left\{\frac{\beta_{1}}{2 \beta_{0}} Y^{2}+\left(\frac{\Gamma_{\text {cusp }}^{(2)}}{\Gamma_{\text {cusp }}^{(1)}}-\frac{\beta_{1}}{\beta_{0}}\right)\left(\frac{\alpha_{s}\left(\mu_{0}\right)-\alpha_{s}(\mu)}{\alpha_{s}\left(\mu_{0}\right)}-Y\right)\right\}+\cdots,
\end{aligned}
$$

where $\beta_{0}=\left(11 C_{G}-2 N_{f}\right) / 3, \beta_{1}=\left(34 C_{G}^{2}-10 C_{G} N_{f}\right) / 3-2 C_{F} N_{f}$ are the first two coefficients of the $\beta$ function, $Y \equiv \ln \left[\alpha_{s}\left(\mu_{0}\right) / \alpha_{s}(\mu)\right]$, and the ellipses are down by $\alpha_{s}$. The Sudakov-type enhancement, induced by $\int_{\alpha_{s}\left(\mu_{0}\right)}^{\alpha} d \alpha^{\prime} / \beta\left(\alpha^{\prime}\right) \sim 1 / \alpha$ in (6), makes the 2-loop cusp anomalous dimension $\Gamma_{\text {cusp }}^{(2)}$ contribute to (7) at the same level as $\gamma_{F}^{(1)}$. We take into account the corresponding effect of $\Gamma_{\text {cusp }}^{(2)}$ according to consistent order counting, i.e., by a systematic "logarithmic expansion" of (5) up to the next-to-leading logarithmic (NLL) accuracy, treating $\left(\beta_{0} \alpha_{s}(\mu) / 2 \pi\right) \ln \left(\mu / \mu_{0}\right)$ as $O(1)$ [9].

Now, we evaluate (3) as the "initial" LCDA at the scale $\mu=1 \mathrm{GeV} \equiv \mu_{0}$, and evolve this result using (5) to higher scale $\mu \sim \sqrt{m_{b} \Lambda_{\mathrm{QCD}}}$, relevant to the factorization formula for exclusive $B$ decays. We calculate (3) and (5) for imaginary light-cone separation, performing the Wick rotation $t \rightarrow-i \tau[7,6]$. For the HQET parameters in (3), we use $\bar{\Lambda}=\bar{\Lambda}_{D A}(\mu)\left[1+(7 / 16 \pi) C_{F} \alpha_{s}\right]-$ $(9 / 8 \pi) \mu C_{F} \alpha_{s}$, with $\bar{\Lambda}_{D A}\left(\mu_{0}\right) \simeq 0.52 \mathrm{GeV}$, and $\lambda_{E}^{2}\left(\mu_{0}\right) \simeq 0.11 \mathrm{GeV}^{2}, \lambda_{H}^{2}\left(\mu_{0}\right) \simeq 0.18 \mathrm{GeV}^{2}$, following our previous work [6]. We obtain model-independent description of the $B$-meson LCDA $\tilde{\phi}_{+}^{\mathrm{OPE}}\left(-i \tau, \mu_{0}\right)$ for $\tau \lesssim 1 / \mu_{0}=1 \mathrm{GeV}^{-1}$, which is displayed by the solid line in Fig. 1 (a). This result can be substituted directly into (5) as the input LCDA for the case with $\tau \lesssim 1 \mathrm{GeV}^{-1}$, because $z \tau \leq \tau \lesssim 1 \mathrm{GeV}^{-1}=1 / \mu_{0}$ in the integrand, reflecting the quasilocal nature. We obtain the 

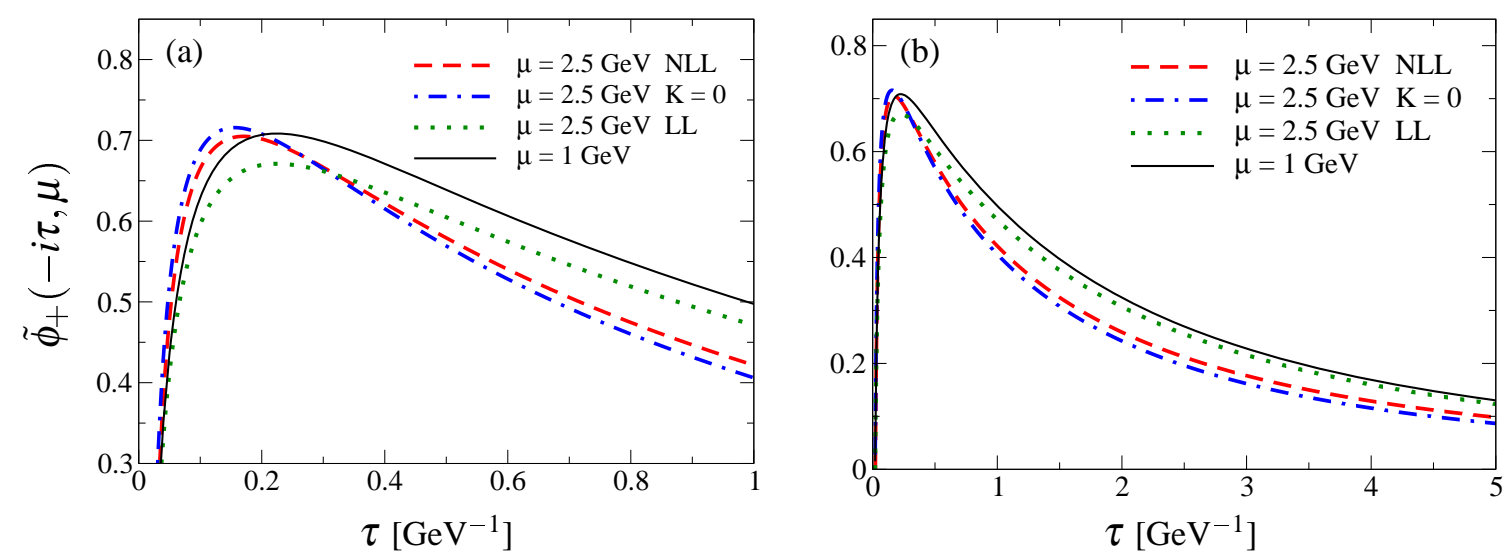

Figure 1: The evolution of the $B$-meson LCDA based on the coordinate-space formula (5) using (a) $\tilde{\phi}_{+}^{\mathrm{OPE}}\left(-i \tau, \mu_{0}\right)$ of (3) and (b) its extension with (8), respectively, as the input DA shown by the solid curve.

dashed line in Fig. 1 (a) as the full result of the LCDA $\tilde{\phi}_{+}(-i \tau, \mu)$ at $\mu=2.5 \mathrm{GeV}$ by our NLL evolution (5). When we omit the term with $K\left(z, \alpha_{s}\right)$ in (4), the resultant evolution yields the dotdashed curve. If we omit the other terms at the NLL level further, involving $\xi \rightarrow 0, \Gamma_{\text {cusp }}^{(2)} \rightarrow 0$, $\gamma_{F}^{(1)} \rightarrow 0, \beta_{1} \rightarrow 0$ in (5), we obtain the dotted curve as the result of the evolution at the LL accuracy. We see the considerable Sudakov suppression not only at the LL level but also at the NLL level; in particular, at the NLL level, the suppression arises in the moderate $\tau$ regions, while the DA is enhanced for small $\tau$. On the other hand, the DGLAP-type splitting function contributes to shifting the distribution from small to moderate $\tau$, as a result of the integral over $z$ in (5). Our full result, the dashed curve, is useful for providing the model-independent behavior of the $B$-meson LCDA in small and moderate $\tau$ regions presented in Fig. 1 (a).

Apparently, the OPE form (3), used for the input DA, breaks down in the large $\tau$ region. We rely on a model function to describe the DA in the large $\tau$ region dominated by the nonperturbative effects, and, specifically, we use the following form of the input DA for the entire range of $\tau$ [6],

$$
\tilde{\phi}_{+}\left(-i \tau, \mu_{0}\right)=\theta\left(\tau_{c}-\tau\right) \tilde{\phi}_{+}^{\mathrm{OPE}}\left(-i \tau, \mu_{0}\right)+\theta\left(\tau-\tau_{c}\right) \frac{N}{\left(\tau \omega_{0}+1\right)^{2}},
$$

with $\tau_{c} \sim 1 / \mu_{0}$, where the second term represents a model for the large $\tau$ behavior, whose simple form was also adopted in [8] as a nonperturbative component to model the $B$-meson LCDA using the information from the OPE with the local operators of dimension $d \leq 4$ and the NLO corrections to the corresponding Wilson coefficients taken into account. (For the difference between our OPE (3) and the OPE derived in [8], see [6].) The two parameters $N$ and $\omega_{0}$ are determined by the continuity of (8) and its derivative at $\tau=\tau_{c}$. The resulting values, $N \simeq 0.86$ and $\omega_{0} \simeq 0.31 \mathrm{GeV}$, are found to be stable under the variation of $\tau_{c}$ for $0.6 \mathrm{GeV}^{-1} \lesssim \tau_{c} \lesssim 1 \mathrm{GeV}^{-1}$, and so is the behavior of the corresponding DA (8) [6]. Taking $\tau_{c}=1 \mathrm{GeV}^{-1}$, the solid curve in Fig. 1 (a) is now continued to the $\tau \geq 1 \mathrm{GeV}^{-1}$ region with (8), as presented by the solid curve in Fig. 1 (b). Using this as the input DA in (5), we get the other curves in Fig. 1 (b), which are evolved in the same way as the corresponding curves in Fig. 1 (a). Note, the behaviors of those new curves in the region $\tau \leq \tau_{c}=1 \mathrm{GeV}^{-1}$ are not contaminated under evolution by the model behavior for larger distances. 


\begin{tabular}{|c|c|c|c|}
\hline & \multicolumn{3}{|c|}{$\lambda_{B}^{-1}(\mu)\left[\mathrm{GeV}^{-1}\right]$} \\
\hline$\mu$ [GeV] & Eq. (9) with Eqs. (5), (8) & Lee-Neubert [8] & Braun et al. [7] \\
\hline 1.0 & $2.7(0.6+2.1)$ & 2.1 & 2.2 \\
1.5 & $2.4(0.6+1.8)$ & 1.9 & \\
2.0 & $2.2(0.5+1.7)$ & 1.7 & \\
2.5 & $2.1(0.5+1.6)$ & 1.6 & \\
\hline
\end{tabular}

Table 1: The inverse moment $\lambda_{B}^{-1}(\mu)$ of (9). The results obtained by other works are also shown.

Integrating the dashed curve in Fig. 1 (b) over $\tau$, we get the first inverse moment of the LCDA,

$$
\lambda_{B}^{-1}(\mu) \equiv \int_{0}^{\infty} d \omega \frac{\phi_{+}(\omega, \mu)}{\omega}=\int_{0}^{\tau_{c}} d \tau \tilde{\phi}_{+}(-i \tau, \mu)+\int_{\tau_{c}}^{\infty} d \tau \tilde{\phi}_{+}(-i \tau, \mu),
$$

at the scale $\mu=2.5 \mathrm{GeV}$, as the sum of the model-independent and -dependent contributions, the first and second terms in the RHS. $\lambda_{B}^{-1}(\mu)$ with $\mu \sim \sqrt{m_{b} \Lambda_{\mathrm{QCD}}}$ arises in the factorization formula for exclusive $B$ decays [1,2]. Table 1 shows the results for $\mu=2.5 \mathrm{GeV}$ and other values of $\mu$, with the first and second numbers in the parentheses denoting the contributions from the first and second terms in (9). The evolution decreases $\lambda_{B}^{-1}(\mu)$, in particular, through the decrease of the model-dependent contribution, and the larger value of $\lambda_{B}^{-1}\left(\mu_{0}\right)$ leads to the larger $\lambda_{B}^{-1}(\mu)$ for $\mu \sim \sqrt{m_{b} \Lambda_{\mathrm{QCD}}}$; as emphasized in [6], our larger $\lambda_{B}^{-1}\left(\mu_{0}\right)$ than the corresponding values of other works $[8,7]$ originates from the novel contribution of $\lambda_{E}^{2}$ and $\lambda_{H}^{2}$ in (3), associated with the dimension-5 three-particle operators.

Finally, we note the simple form of our solution (5) allows us to express (9) compactly as [9]

$$
\lambda_{B}^{-1}(\mu)=\frac{e^{\mathscr{V}\left(\mu, \mu_{0}\right)+\left(1-\gamma_{E}\right) \xi}}{\Gamma(1+\xi)} \int_{0}^{\infty} d \tau\left(\tau \mu_{0} e^{\gamma_{E}}\right)^{-\xi} \tilde{\phi}_{+}\left(-i \tau, \mu_{0}\right) .
$$

Combined with the $\tau \rightarrow 0$ and $\tau \rightarrow \infty$ behaviors of $\tilde{\phi}_{+}\left(-i \tau, \mu_{0}\right)$, as indicated respectively by (3) and [3], this integral proves to converge for $\xi<1$, which is satisfied for all relevant cases.

\section{References}

[1] M. Antonelli et al., arXiv:0907.5386 [hep-ph], and references therein.

[2] M. Beneke and S. Jager, Nucl. Phys. B751 (2006) 160; B768 (2007) 51; V. Pilipp, Nucl. Phys. B794 (2008) 154; G. Bell, B822 (2009) 172; G. Bell and V. Pilipp, Phys. Rev. D80 (2009) 054024.

[3] A. G. Grozin and M. Neubert, Phys. Rev. D55 (1997) 272; H. Kawamura, J. Kodaira, C.F. Qiao and K. Tanaka, Phys. Lett. B523 (2001) 111; Erratum-ibid. B536 (2002) 344.

[4] B. O. Lange and M. Neubert, Phys. Rev. Lett. 91 (2003) 102001.

[5] G. Bell and T. Feldmann, JHEP 0804 (2008) 061; S. Descotes-Genon and N. Offen, JHEP 0905 (2009) 091.

[6] H. Kawamura and K. Tanaka, Phys. Lett. B673 (2009) 201.

[7] V. M. Braun, D. Y. Ivanov and G. P. Korchemsky, Phys. Rev. D69 (2004) 034014.

[8] S. J. Lee and M. Neubert, Phys. Rev. D72 (2005) 094028.

[9] H. Kawamura and K. Tanaka, arXiv:1002.1177 [hep-ph]. 